ReVista de Filosofía

Volumen 71 (2015) 151-162

\title{
CONSIDERACIONES SOBRE EL INFINITO EN LA FILOSOFÍA DE KANT
}

\author{
Diego Sanhueza* \\ Pontificia Universidad Católica de Valparaíso \\ pielago123@gmail.com
}

\begin{abstract}
Resumen / Abstract
En este artículo se analiza la noción de infinito en la filosofía de Kant. Este análisis es pertinente y necesario porque Kant, en relación con este tema, realiza afirmaciones que parecen ser abiertamente contradictorias. Por el contrario, en este artículo se defenderá la idea de que hay una noción clara y consistente de lo infinito, y esto no solo en la Crítica de la razón pura, sino también en obras anteriores y posteriores.
\end{abstract}

PALABRAS ClAVE: infinito, indeterminado, cualidad, cantidad, síntesis.

\section{CONSIDERATIONS ON INFINITY IN THE PHILOSOPHY OF KANT}

In this article, the notion of infinity is analyzed in the philosophy of Kant. This analysis is relevant and necessary for Kant, on this subject, makes statements that seems to be openly contradictory. By contrast, in this article is defend the idea that there is a clear and consistent notion of infinity, and this not only in the Critique of Pure Reason, but also in earlier and later works.

KEY WORDS: infinity, indeterminate, quality, quantity, synthesis.

\section{Introducción}

Como lo han notado varios comentaristas, la noción de infinito presenta
algunas dificultades en el pensamiento de Kant. Si nos remitimos al contexto
de la Crítica de la razón pura, resulta que el modo en que esta noción es tratada en la
Estética y el modo en que lo es en la Dialéctica parecen ser incompatibles. Sin ir más
lejos, Cohen ha dicho: "la contradicción debe resultar chocante para todo conocedor de
la doctrina de las Antinomias" (Cohen 1925, 119). Y, en efecto, podemos ver que Kant
asevera, por un lado, que la noción de "infinito" puede ser legítimamente predicada

Profesor de Filosofía de la Universidad de Chile. Doctorando en Filosofía de la Pontificia Universidad Católica de Valparaíso. 
de la intuición pura en la primera de estas secciones y, por otro lado, que la noción de infinito es abiertamente calificada de imposible en la segunda. Para lo primero: "El espacio es representado como una magnitud infinita dada" (B39) ${ }^{1}$. Para lo segundo: "Tal concepto de cantidad, como infinitud dada, es empíricamente imposible, y por tanto es absolutamente imposible también con respecto al mundo [entendido] como objeto de los sentidos" (A520/B548). ¿Existe, pues, una dificultad real en relación con la noción de infinito en el pensamiento de Kant?

En lo que sigue, intentaremos una reconstrucción de los argumentos referidos a la noción de infinito. Nuestra pregunta guía será la siguiente: ¿qué se debe entender por "infinito" en la filosofía crítica de Kant y, en especial, en la KrV? Haremos primero una reconstrucción cronológica que no se limitará a la $\mathrm{KrV}$ y luego, al final, aunaremos los resultados.

\section{El infinito en la Dissertatio de 1770}

a) La noción general de infinito

En una nota a pie de página del primer parágrafo de los Principios formales del mundo sensible y del inteligible ${ }^{2}$, Kant tematiza la noción de infinito. En primer lugar, expone una "falsa" noción de éste, según la cual significa tanto como una magnitud (quantum, Grösse) mayor que la cual toda otra es imposible (Dissertatio, 5. AA II, 388). Una definición semejante, que homologa lo infinito con lo "máximo" o un "número infinito", se puede fácilmente refutar: si el infinito es una magnitud determinada o un número, siempre habrá una cantidad o número mayor. ¿Por qué? Porque todo número puede siempre ser aumentado por adición de otra unidad. Además, esta definición contiene en sí misma una contradicción, pues un número no puede sino constar de un número finito de unidades. Y tal es precisamente el modo en que argumentan los detractores del infinito. Sin embargo, a ojos de Kant, ellos solo pelean contra una falsa noción del infinito.

Por el contrario, la verdadera noción de infinito podría formularse así: una multitud (multitudo, Menge) mayor que todo número (Dissertatio, 5. AA II, 388). Como se puede apreciar, la perspectiva es completamente distinta. El infinito no es identificado con un número infinitamente grande, sino con aquello que excede todo número. Cabe preguntarse entonces qué sea un número y cómo el infinito podría "excederlo". En la línea siguiente, Kant ofrece algunas indicaciones al respecto: "Sólo se puede llegar al concepto determinado de multitud añadiendo sucesivamente unidad a unidad y sólo

1 En adelante, citaremos como KrV. Los ocasionales paréntesis cuadrados corresponden a agregados del traductor.

2 En adelante, citada como Dissertatio. 
se puede llegar al concepto completo llamado número, realizando esa progresión en un tiempo finito" (Dissertatio, 5. AA II, 388). Según esto, el número es el resultado de la sucesiva adición de unidades hasta un determinado fin. El lector familiarizado con el capítulo del Esquematismo de la $\mathrm{KrV}$ no podrá dejar de ver en esta caracterización un antecedente de la noción de número que, en tanto esquema de las categorías de la cantidad, es descrito como "representación que abarca la sucesiva adición de lo uno a lo uno (homogéneo)" (A142/B182).

Tomando en consideración esto, ¿en qué sentido, entonces, el infinito excede al número? El infinito es aquella materia cuantificable que el proceso de adición supone para su ejercicio, pero que no puede ser agotada mediante tal ejercicio. Infinito es, pues, un predicado que se aplica a una cantidad indeterminada, que es determinable por síntesis (adición de unidades), pero que, al mismo tiempo, permanece al margen de ésta como un resto estructuralmente indeterminable.

b) El infinito cualitativo y el cuantitativo

La referencia a la noción de número que acabamos de hacer nos indica que el examen kantiano de la noción de infinito estará preferentemente orientado por una categoría específica, la de la "cantidad". Sin embargo, también debemos reconocer que esta noción se puede aplicar a otra categoría, la de la cualidad. Así, el infinito kantiano puede ser entendido en dos dimensiones y éstas implican dos lógicas distintas. Por un lado, cuantitativamente hablando, se tratará de un infinito en el progreso de las partes hacia el todo y le corresponderá una acción denominada síntesis. Por otro lado, cualitativamente hablando, se tratará de un infinito en el regreso del todo hacia sus partes y le corresponderá una acción denominada análisis (cfr. primer parágrafo de la Dissertatio). Revisemos con un poco más de detalle cada una de estas dimensiones.

Al menos en dos oportunidades, pasando revista a las propiedades que le conciernen a la intuición pura en cuanto tal, Kant asevera que la "continuidad" es una de estas propiedades (cuarto argumento del parágrafo 14 dedicado al tiempo y nota a pie de página del parágrafo 15 dedicado al espacio). La continuidad es una propiedad cualitativa que debe ser entendida del siguiente modo: "cuanto que no consta de partes simples" (Dissertatio, 18. AA II, 399). Si una representación es continua y no tiene partes simples, entonces el análisis (división, regresión hacia la parte) se ejerce infinitamente. ¿Por qué? Porque precisamente nunca encontrará un término definitivo, una parte simple. Kant lo afirma explícitamente: "En el cuanto continuo, el regreso desde el todo a la parte carece de término (...) y, por lo tanto, el análisis completo es imposible" (Dissertatio, 5. AA II, 388). Dicho de otro modo, "infinitud" es un predicado que se le puede asignar a la intuición en la medida en que ésta es continua, es decir, en la medida en que el número ya contabilizado de sus partes presupone una cantidad indeterminada de partes que aún (y siempre) pueden ser producidas por análisis.

En lo que respecta al examen cuantitativo, atendamos a la siguiente cita: 
Solamente si se dan un espacio y un tiempo infinitos es designable por limitación cualquier espacio o tiempo determinado, y ni el punto ni el momento pueden ser pensados por sí mismos, sino solamente pueden ser concebidos en un espacio y en un tiempo ya dados, como términos (termini, Grenzen) suyos (Dissertatio, 24. AA II, 405).

La primera parte de la cita señala que la intuición pura infinita es condición de posibilidad de la parte. La caracterización es correcta y, no obstante, resulta un poco difícil discernir entre la lógica cuantitativa y la cualitativa. En efecto, ¿no era éste exactamente el mismo argumento esgrimido en el análisis cualitativo? Esta impresión se refuerza cuando, en esta cita, leemos que la parte se obtiene por limitación del todo. ¿Qué diferencia hay entre esta limitación cuantitativa y el análisis cualitativo que va del todo a sus partes?

La diferencia se puede apreciar cuando reparamos en la peculiar naturaleza de esta limitación. Ésta debe ser entendida como una "síntesis" y no como un "análisis". Pues bien, ¿qué es lo sumado-sintetizado en este caso? Lo refiere la segunda parte de la cita: puntos y momentos, vale decir, "términos" o "límites". La síntesis suma términos o límites, y ésta es la razón por la cual la síntesis cuantitativa se resuelve paradójicamente en una limitación de la intuición pura. Como indica la cita, esta limitación tiene como resultado una intuición pura determinada (=numerada), mas presupone la donación de momentos y puntos. Ahora bien, éstos, en tanto que límites, presuponen a su vez el todo limitable de la intuición pura: "ni el punto ni el momento pueden ser pensados por sí mismos, sino solamente pueden ser concebidos en un espacio y en un tiempo ya dados" (Dissertatio, 24. AA II, 405). La síntesis que suma "términos" no se topa jamás con un término absoluto (pues siempre quedan más instantes y más puntos en la cantidad indeterminada, infinita) y esto significa que ella se ejecuta infinitamente: "En lo infinito, el progreso de las partes hacia el todo carece de término (...) y, por lo tanto, la síntesis completa es imposible" (Dissertatio, 5. AA II, 388). En definitiva, diremos que la intuición pura es infinita cuantitativamente porque el número de partes ya determinadas nunca coincide con la cantidad indeterminada de la intuición pura total.

De una revisión cuidadosa de la Dissertatio se obtiene, por lo tanto, los siguientes resultados. En primer lugar, hay que saber distinguir entre una correcta noción de infinito y una incorrecta. Y, en segundo lugar, esta noción correcta de infinito puede ser entendida tanto cuantitativa como cualitativamente.

\section{Crítica de la razón pura}

Existen dos momentos en la Crítica de la razón pura en que Kant analiza la idea de lo infinito. En primer lugar, en la Estética trascendental, cuarto argumento de la Exposición metafisica del espacio y quinto argumento de la del tiempo (B40 y B48, respectivamente). En segundo lugar, en las observaciones a la Primera antinomia (A432/B460). Aquí, en la Dialéctica, Kant expone el concepto general de lo infinito de modo similar al que lo hizo en la Dissertatio y, por ello, lo utilizarnos como pivote para pasar luego al examen de la Estética. 
a) Primera antinomia: concepto general de lo infinito

Efectivamente, el modo en que Kant presenta la noción de infinito en la Primera Antinomia es bastante similar al modo en que lo hizo en la Dissertatio. Aquí también parte enunciando el "falso" concepto de infinito: "Infinita es una magnitud (Größe) [tal], que no es posible otra mayor que ella" (A431/B459). E inmediatamente agrega: "ese concepto no concuerda con lo que se entiende por un todo infinito" (Ibíd.). En el párrafo siguiente, Kant ofrece la noción correcta: "El concepto verdadero (trascendental) de la infinitud es: que la síntesis sucesiva de la unidad en la medición de un quantum nunca puede ser acabada" (A432/B466). Si prestamos atención, esta definición general parte enunciando aquello que concierne a la síntesis: ésta se ejecuta infinitamente. Vale decir, la infinitud no es concebida en primer lugar como predicado de la intuición, sino de la síntesis (volveremos sobre esto un poco más adelante, al introducir una diferencia entre un infinito potencial y uno actual). Sin embargo, exactamente en el mismo lugar, aunque esta vez en una nota a pie de página, Kant retoma la idea de que la noción de "infinito" puede predicarse de la cantidad intuitiva. Anota: "Este [quantum] contiene en sí una multitud (Menge) (de la unidad dada) que es mayor que cualquier número" (A432/B460).

Este infinito mayor que todo número es el único que se acepta como legítimo en el marco de la filosofía trascendental kantiana. Es, pues, sobre el otro concepto de infinito - un concepto de infinito determinado, numerado, cuantificado- que giran todas las críticas de la filosofía kantiana, y el que ha generado algunos malentendidos. En este sentido, se puede revisar con sumo provecho los argumentos que la tesis ejerce en contra de la antítesis en la primera antinomia (la antítesis sostiene precisamente la infinitud del mundo).

b) Estética: infinito cualitativo y cuantitativo

Tanto el cuarto argumento de la Exposición metafísica del espacio como el quinto de la del tiempo abordan la infinitud de la intuición pura. El primero de ellos se desenvuelve bajo una perspectiva cualitativa y el del tiempo, bajo una cuantitativa. Partamos con el argumento cualitativo del espacio ${ }^{3}$.

Kant parte enunciando la tesis del infinito: "El espacio es representado como una magnitud infinita dada" (B39). El razonamiento transcurre al modo de una comparación entre el concepto y la intuición, comparación relativa a la manera en que cada una de estas representaciones contiene una cierta infinitud. El concepto, sostiene Kant, contiene una infinitud bajo sí, pues el concepto es en sí mismo la "característica común" que se le puede aplicar a infinitos objetos que están subordinados como sus casos (el concepto "hombre" tiene bajo sí una infinita cantidad de hombres concretos, singulares). En

3 Para la diferencia entre estos dos tipos de infinito en el contexto de la $\mathrm{KrV}$ véase: Vaihinger (1892) 221-223 y 253. También: Falkenstein (1995) 231-232 y 237. 
cambio, la intuición pura contiene en sí una cantidad infinita de representaciones coordinadas. Esto significa que, cualitativamente hablando, la división del espacio es un regressus infinito porque no se puede encontrar jamás una parte simple en él: "Todo espacio intuido en sus límites es un todo tal, cuyas partes, en toda descomposición, son siempre, a su vez, espacio y por eso divisible al infinito" (A524/B552). Esto implica necesariamente que al espacio le corresponde la propiedad de ser un quantum continuum (cfr. A169/B211).

El argumento cuantitativo relativo a la infinitud del tiempo aparece formulado del siguiente modo:

La infinitud del tiempo no significa nada más, sino que toda cantidad determinada de tiempo es posible solo mediante limitaciones de un tiempo único que sirve de fundamento. Por eso, la representación originaria del tiempo debe ser dada como ilimitada (B47-48).

En este caso, la noción de "limitación" (Einschränkung) juega nuevamente un rol clave en el argumento. Como vimos en el apartado anterior, ésta debe ser entendida como una síntesis que suma límites o términos (Grenze, A169/B211) para obtener como resultado un tiempo determinado (=número). Por ejemplo, así es como Kant describe la determinación del tiempo en los Axiomas de la intuición: "En él [el tiempo] pienso solamente el transito sucesivo de un instante a otro, donde, a través de todas las partes del tiempo, y de su agregación, se genera una magnitud de tiempo determinada" (A163/B203). Este tiempo determinado es una parte del tiempo total, el cual, como tal, permanece como un fundamento indeterminado (=infinito) y hace de la síntesis un proceso sin término absoluto.

\section{c) Resumen}

Antes de pasar a la siguiente sección, resumamos brevemente lo que hemos visto en el examen de la KrV. En primer lugar, al igual que en la Dissertatio, también podemos apreciar la distinción entre un infinito bien entendido y un infinito mal comprendido (=máximo). Tener claridad sobre esta distinción es clave para entender el hecho de que Kant, por un lado, acepte el predicado (trascendental) de infinito en relación con la intuición pura y, por otro lado, niegue que se pueda conocer la infinitud del mundo (antítesis de la primera Antinomia). En segundo lugar, el infinito bien comprendido

4 Toda representación intuitiva, por el solo hecho de ser tal, es continua. Esto significa que ni la intuición pura ni la intuición empírica contienen partes simples. Sin embargo, esta "infinitud" de partes se realiza de un modo muy distinto en el caso de la intuición pura y en el caso de la empírica. En relación con la primera, cabe decir que la parte de la intuición pura es homogénea con el todo: una parte del espacio es tan espacial como el espacio mismo. Por el contrario, esto no se cumple en relación con la segunda: una parte de una mesa ya no es una mesa. 
también se puede entender tanto cualitativa como cuantitativamente. En el primer caso, se trata de la propiedad de la continuidad (inexistencia de parte simple). Y ahora podríamos agregar: toda representación intuitiva, por el hecho de serlo, es continua (para las diferencias entre la intuición empírica y pura, véase nota 12 de este trabajo). En el segundo caso, solo la intuición pura es cuantitativamente infinita. La intuición empírica, por el contrario, se define por tener límites cuantitativos. Kant indica, por ejemplo, que el fenómeno externo "está encerrado entre límites (cuerpo)" (A525/ B553) $)^{5}$. Esto significa que, en el caso de la intuición empírica, la síntesis encuentra un límite absoluto, se detiene y conforma un todo. Nada de ello ocurre con la intuición pura: aquí la síntesis progresa de límite relativo a límite relativo (puntos e instantes), no se encuentra jamás con un límite absoluto y, por lo tanto, no conforma el todo de la intuición pura. Ésta es cuantitativamente infinita, entonces, en el sentido de lo indeterminado o, mejor dicho, lo indeterminable.

Me parece que las razones que acabamos de exponer dan cuenta de una noción perfectamente consistente de lo infinito en el marco de la KrV. Como hemos tenido oportunidad de apreciar, esta concepción se deriva, en lo fundamental, de la Dissertatio $\mathrm{y}$, como veremos a continuación, se conserva en los escritos críticos que vendrán posteriormente.

\section{El ensayo sobre Kästner}

El ensayo sobre los artículos de Kästner ${ }^{6}$ fue escrito en 1790 en el marco de una polémica con Eberhard, y su redacción coincide con la publicación del escrito Über eine Entdeckung nach der alle neue Kritik der reinen Vernuft durch eine ältere entbehrlich gemacht werden soll, que precisamente pretendía zanjar esa disputa. No fue publicado en vida de Kant, sino años más tarde por Dilthey (1889). Pues bien, ocurre que, en el segundo tomo de la revista Philosophisches Magazin (1790), Eberhard publicó tres artículos del matemático alemán Kästner con el objeto de demostrar que la palabra del matemático le daba la razón a él (Eberhard) y no a Kant. Muy por el contrario, nuestro filósofo redacta este ensayo convencido de que estos artículos hablan irrefutablemente a favor de la postura crítica.

5 También, una definición de cuerpo: "materia dada dentro de sus límites" (A513/B541). Ello significa que la intuición empírica es cualitativamente infinita (cantidad infinita, indeterminada de partes) y cuantitativamente finita. A ello se refiere Kant cuando escribe lo siguiente: "Podemos intuir como un todo un quantum indeterminado, cuando está encerrado dentro de límites (...). Pues los límites determinan ya la integridad, al excluir todo aditamento" (A427-428/B455-456).

$6 \quad$ AA. XX, Über Kästners Abhandlungen, pp. 410-423. No existe traducción al español de este escrito, por lo que las traducciones que aparecen en este escrito son mías. Los paréntesis cuadrados son agregados del traductor. 
Kant dedica la segunda mitad del ensayo sobre Kästner a dilucidar la noción de infinito. A su parecer, esta aclaración se hace necesaria porque tanto la metafísica como la geometría se ocupan de lo infinito, aunque ambas de modo diferente: "pertenece a la recensión de una revista que ha hecho de la metafísica su objetivo principal [la Philosophisches Magazin], hacer reconocible la diferencia del uso del concepto de lo infinito en la metafísica y en la geometría" (AA XX, 417-418).

Para introducir el asunto de lo infinito, Kant recurre al ejemplo de la línea que puede ser trazada infinitamente: “¿Qué significa esto: que uno siempre pueda trazar una línea recta más allá de lo que ésta ya ha podido ser trazada?" (AA XX, 418). La respuesta es la siguiente: "Significa que el espacio en el cual yo describo una línea es más grande que todo espacio que yo pueda describir en él" (Ibíd.). Como vemos, estas afirmaciones calzan totalmente con lo que hemos examinado anteriormente: el espacio es un quantum más grande que la línea que puede ser trazada en él o, lo que es lo mismo, más grande que el espacio que puede ser mensurado mediante un número.

Sin embargo, con esto no hemos respondido aún la pregunta relativa a la diferencia entre el modo en que la geometría y la metafísica conciben lo infinito. ¿Cuál es esta diferencia? En la página 419, Kant realiza un interesante contrapunto entre la metafísica y la geometría. Anota: "En aquella [la metafísica], el espacio es considerado como dado antes de toda determinación según un cierto concepto de objeto; en ésta [geometría], es hecho. En aquella, él es originario y solo un (único) espacio; en ésta, es derivado y hay allí (muchos) espacios". Según esto, la metafísica se ocupa de un espacio preconceptual y, en esa misma medida: dado, originario y único. Por su parte, la geometría trabaja sobre una intuición descrita y, en esa misma medida: hecha, derivada y múltiple. En consecuencia, "el espacio geométrico y objetivamente dado siempre es finito" (AAXX, 419), donde finitud equivale a "estar limitado", vale decir, estar determinado según concepto; por el contrario, el espacio metafísico-originario (a saber: preconceptual) es infinito (AA XX, 420-421). No obstante, de aquí no se debe concluir que solo la metafísica se vincula con lo infinito, pues Kant ha señalado expresamente que tanto una disciplina como la otra lo hacen. Entonces, ¿qué tipo de vínculo tiene la geometría con lo infinito? La respuesta consiste en indicar que el espacio de la geometría si bien es finito, contiene sin embargo límites que no son absolutos y, por lo tanto, habría un infinito potencial en todo espacio finito (cfr. AA XX, 412 y 418). Ahora bien, este infinito potencial es lo que debemos identificar con la infinitud de una acción que se ejecuta sin término. Por el contrario, la metafísica se vincula con un infinito actual (cfr. AA XX, 418 y 421), el cual es la condición de posibilidad del potencial: "este infinito potencial, que el matemático necesariamente tiene como fundamento de sus determinaciones del espacio, presupone una infinitud actual (mas solo metafísica) y efectivamente real y solo es posible bajo esta presuposición" (AA XX, 418) ${ }^{7}$.

7 En este punto, conviene aclarar que, por expresa indicación de Kant, si bien estas observaciones se ciñen exclusivamente al espacio, pueden sin embargo ser aplicadas legítimamente al tiempo. Cfr. AA XX, 419. 
No deja de ser importante insistir una vez más en el modo correcto en que debe ser entendida la relación entre el infinito potencial de la síntesis y el infinito actual de la representación intuitiva: la síntesis es potencialmente infinita porque la intuición pura es actualmente infinita, y no al revés: la intuición pura no es infinita por la acción infinita de la síntesis, esto es, el infinito intuitivo indeterminable no es producido por síntesis.

\section{El infinito en la $K U$}

Los desarrollos de la Crítica del juicio $^{8}$ relacionados con la noción de infinito difieren bastante de lo que hemos señalado hasta el momento. La razón de ello es clara: en este texto, a Kant le interesa el rendimiento estrictamente subjetivo de esta noción, es decir, su conexión con el sentimiento de lo sublime. Sin embargo, el filósofo se sirve exactamente de la misma noción (trascendental) de infinito que hemos revisado hasta el momento, y por esta razón su exposición reviste interés para nosotros. En lo que sigue, nos limitaremos a aquello que Kant denomina sublime matemático (parágrafos 25 y 26).

A lo primero que debemos atender es a una distinción que Kant establece entre una estimación lógica de magnitudes y una estimación estética. La primera corresponde a un juicio determinante y se sirve de las categorías de la cantidad. La segunda, en cambio, corresponde a un juicio reflexionante y, en consecuencia, carece de concepto. Por medio de la primera podemos determinar el número exacto de una magnitud determinada, mientras que por la segunda podemos saber si algo es "grande" o "pequeño" en general ( $\sin$ concepto, sin una cifra exacta). Pues bien, existe una diferencia fundamental entre una y otra estimación, y esta diferencia concierne al límite. La estimación lógica carece de un máximo y puede ejecutarse infinitamente: no habrá jamás un número que sea más grande que el resto. Por el contrario, la comprensión estética posee un máximo, un límite. Así, pues, puede ocurrir que un objeto X sea demasiado grande como para que su magnitud sea estéticamente comprendida por el sujeto. Pues bien, argumenta Kant, este fracaso en la comprensión estética está en el origen del sentimiento de lo sublime. Sin embargo, aún es insuficiente como para explicarlo satisfactoriamente. ¿Cómo es, entonces, que esta experiencia negativa de la imposibilidad de mensurar un objeto empírico se transforma en la experiencia positiva de un sentimiento de lo sublime? Por la intervención de la razón, aduce Kant. Estas son sus palabras:

El ánimo escucha en sí la voz de la razón, que exige totalidad para todas las magnitudes dadas, incluso para aquellas que, ciertamente, nunca pueden aprehenderse totalmente, pero que al mismo tiempo (en la representación sensible) se enjuician como totalmente dadas. En esta medida, exige comprensión en una intuición y reclama una exhibición (Darstellung) de todos aquellos miembros de una serie numérica que crece progresivamente. Ni tan siquiera lo infinito (espacio y tiempo

8 En adelante, citada como KU. 
expirado) se exceptúa de esta exigencia, sino que más bien se hace inevitable pensarlo (en el juicio de la razón común) como totalmente dado (según su totalidad) (KU, 211. AA V, 254).

Entonces: por obra de la razón, la comprensión estética toma conocimiento de lo infinito, despertando con ello el sentimiento de lo sublime. ¿En qué sentido? Ya sabemos que, con ocasión de un objeto excesivamente grande, la imaginación llega a su límite y no puede mensurar Pues bien, la razón -por decirlo así-fuerza a la imaginación a que traspase dicho límite y a que intente, pese a todo, comprender el todo del objeto que se le escapa. Como semejante intento está fuera de las capacidades de la imaginación, entonces ésta, razón mediante, intenta atisbar una magnitud "fuera de toda medida". Dicho de otro modo, la razón obliga a que la imaginación lleve a cabo una imposible "exhibición de lo infinito" (KU, 236. AA V, 274) en un objeto empírico. De esta manera, el objeto que ha sobrepasado el límite de la comprensión estética no solo es declarado "irrepresentable" (por estar fuera de la medida), sino que, precisamente por exceder toda medida, es tenido por "infinito". En este sentido, el predicado de "infinito" que le concierne al objeto empírico significa exactamente lo mismo que el predicado de "infinito" aplicado a la intuición pura: algo absolutamente grande, fuera de la medida. En el contexto de la KU, Kant define del siguiente modo la noción de infinito: "lo infinito es lo absolutamente grande (schlechthin gro $\beta$ ) (no de manera meramente comparativa). Comparado con él todo lo demás (magnitudes de la misma especie) es pequeño" (KU 212. AA V, 254) ${ }^{9}$. Por cierto, que el objeto sea infinitamente grande no significa que sea el objeto más grande del universo, sino que, bajo ciertas circunstancias, ha sobrepasado la medida de la apreciación estética. Ahora bien, este objeto infinitamente grande es el que desencadena el sentimiento de lo sublime, pues, en palabras de Kant, "llamamos sublime a aquello que es absolutamente grande (schlechthin groß)" (KU, 203. AA V, 248).

Acabamos de ver en qué sentido la noción de infinito está imbricada en el sentimiento de lo sublime. Sin embargo, aún debemos responder la pregunta más importante: ¿Puede ser considerado "trascendental" este infinito? Una de las notas principales del infinito entendido trascendentalmente es que debe ser concebido de modo indeterminado: el infinito no es un máximo, sino una cantidad mayor que todo número. En el caso de la $\mathrm{KU}$, dado que ya no se trata de la estimación lógica de cantidades, sino de la estimación estética, el infinito ya no podrá ser definido recurriendo a un desmarque en relación con el número. Sin embargo, a su modo, también se trata de un infinito indeterminado (esto es lo importante). En efecto, el objeto sublime es un objeto infinitamente grande en el sentido de que excede toda medida de la estimación estética. Kant es bastante claro en

9 Aquí debemos hacer una aclaración en relación con la traducción. En esta ocasión, Aramayo y Mas han vertido el vocablo "schlechthin" por "sin más ni más". Amén de no ser una traducción muy elegante, presenta el problema de que no se corresponde con el modo en que ellos mismos traducen este vocablo en relación con lo sublime: "grande sin más" (KU, p. 203). Nosotros, siguiendo en esto a las traducciones de Morente y de Oyarzún, hemos puesto "absolutamente" tanto en el caso de lo sublime como en el caso de lo infinito. 
señalar que aquí la exhibición del infinito es "meramente negativa" (KU, 236. AA V, 274): no hay una grandeza infinita determinada, sino indeterminada.

\section{Conclusión}

Retornemos a la pregunta guía que hemos formulado en la introducción: ¿qué se debe entender por "infinito" en la filosofía de Kant? Según lo que hemos visto, podemos aseverar que la noción de infinito puede ser, en primer lugar, entendida correcta o incorrectamente. Entendida de modo correcto, esta noción significa que hay una cantidad que excede a toda estimación de magnitud. Según pudimos apreciar, esta estimación puede ser ya lógica, ya estética, y en ambos casos lo infinito significa lo mismo: un exceso, una indeterminabilidad en relación con el orden de la imaginación (para el caso específico de la KU, véase el capítulo anterior. No insistiremos más sobre sus peculiaridades). En segundo lugar, esta noción correcta de infinito puede ser entendida de dos modos: por un lado y de modo preponderante, es predicado de la representación intuitiva (infinito actual); por otro lado y de modo derivado, es predicado de la acción sin término que se ejecuta sobre la representación intuitiva (infinito potencial). En tercer lugar, cabe consignar que este correcto infinito, entendido de dos modos, puede a su vez ser aplicado a dos dimensiones distintas: la cuantitativa y la cualitativa. En el primer caso, lo infinito actual es predicado exclusivo de la intuición pura e implica un infinito potencial entendido al modo de una síntesis que nunca puede dar con un límite absoluto. En el segundo caso, el infinito actual se predica de la representación intuitiva como tal (continuidad) e implica un infinito potencial entendido al modo de un análisis que nunca encuentra una parte simple.

De esta manera, me parece que se puede demostrar que la noción de infinito en la filosofía de Kant es perfectamente consistente, y que esta consistencia no solo se limita al contexto de la $\mathrm{KrV}$, sino que se extiende tanto a obras precríticias como a obras escritas al inicio de la década de los noventa.

\section{Referencias bibliográficas}

\section{PRINCIPAL}

Kant, Immanuel (1903-1911), Kants gesammelte Schriften. Berlin: Königlich Preussische Akademie der Wissenschaften. Ahora también: (1968- ) Kants Werke. Akademie Textausgabe. Berlin: Walter de Gruyter.

\section{TRADUCCIONES}

Kant, Immanuel (2010), Crítica de la razón pura. Ciudad de México: Fondo de Cultura Económica. Traducción de Mario Caimi. 
(2003), Crítica del discernimiento. Madrid: Antonio Machado Libros. Traducción de R. Aramayo y Salvador Mas.

(1996), Principios formales del mundo sensible y del inteligible (Disertación de 1770). Madrid: Consejo Superior de Investigaciones Científicas. Traducción de Ramón Ceñal Lorente. Edición de José Gómez Caffarena.

\section{SECUNDARIA}

Cohen, Hermann (1925), Kants Theorie der Erfahrung. Berlin: Bruno Cassirer Verlag. Falkenstein,Lorne (1995), Kants Intuitionism. Toronto: University of Toronto Press.

Vaihinger, Hans (1892), Commentar zu Kants Kritik der reinen Vernunft, Band II. Stuttgart/Berlin/Lepzig:Union Deutsche Verlaggeselschaft. 